\title{
Flow Velocity and Effective Viscosity of a Fluid Containing Rigid Cylindrical Inclusions
}

\author{
Siegfried Hess \\ Institut für Theoretische Physik, Technische Universität Berlin, PN 7-1, Hardenbergstr. 36, \\ D-10623 Berlin, Germany
}

Reprint requests to Prof. S. H.; E-mail: S.Hess@physik.tu-berlin.de

Z. Naturforsch. 60a, 401 - 407 (2005); received January 20, 2005

\begin{abstract}
The determination of the flow properties of a fluid containing a cylindrical inclusion with its long axis oriented parallel to the vorticity direction is a 2-dimensional problem which is treated as a special case in a calculation of the corresponding $D$-dimensional problem. The velocity and pressure are obtained from the solution of the equations of hydrodynamics where $D$-dimensional multipole potential tensors are used. The effective viscosity of a dilute suspension is evaluted via the entropy production, as suggested by Einstein, and via an effective stress tensor. The relative change of the viscosity is proportional to the volume fraction. For $D=2$ the proportionality factor $Z$ is found to be 2 and 3 when the inclusion rotates with an angular velocity equal to the vorticity and when the inclusion does not rotate, repectively. The corresponding results for $D=3$ are the well known number $Z=2.5$ and $Z=4$.
\end{abstract}

Key words: Effective Viscosity; Dispersion Rheology; Rigid Inclusion.

\section{Introduction}

Rigid inclusions perturb the flow of a fluid. The analysis of the inclusion-induced modification of the flow properties and in particular the calculation of an effective viscosity are a subject of long standing scientific interest [1-4]. At the same time it is of technical importance, e.g. for the flow of supensions and for the plastic flow of metals as it occurs in connection with dry friction [5]. The performance of brakes [6] as well as the deformation and wear of rails in the railwheel contact zone [7] are strongly affected by rigid inclusions. Here hydrodynamic calculations are presented for the distorted velocity and pressure. Both rotating and non-rotating inclusions are considered, the latter case is typical for dry friction. Special attention is paid to the two-dimensional problem as realised either for a long cylinderical inclusion oriented perpendicular both to the directions of the velocity and its gradient or for a circular inclusion in a surface flow. In order to compare with the well-known results for the three-dimensional case, the calculations are performed for a $D$-dimensional problem where it is understood that the physically relevant cases are $D=2$ and $D=3$. The effective viscosity of dilute suspensions is evaluted via the entropy production as originally suggested by Einstein almost a century ago [1] and via an effective stress tensor, similar to the method used by Landau and Lifschitz [3]. The present study was motivated by Smoothed Particle Dynamics simulations of a two-dimensional multi-inclusion problem with a nonNewtonian fluid matrix [8]. In the limiting case of a dilute system with a Newtonian fluid the numerical results can be compared with the analytic expressions.

This article proceeds as follows. In Section 2 the basic equations and the boundary conditions are stated. The velocity field and the pressure for the $D$-dimensional problem are obtained in terms of $D$-dimensional multipole potential tensors. The case $D=2$ is considered in more detail. Section 3 is devoted to the calculation of the effective viscosity of a dilute suspension. The two methods are employed, viz. routes via the entropy production and via an effective stress tensor. Some concluding remarks are made in Section 4.

\section{Distorted Velocity Field}

\subsection{Formulation of the Problem}

A flow of an incompressible fluid is considered where the gradient of the velocity is constant in the absence of an inclusion. An example is a plane Couette flow where a rigid cylinder with radius $R$ perturbs the velocity field $\boldsymbol{v}$. The long axis of the cylinder is as- 
sumed to be parallel to the vorticity direction, hence one is dealing with a 2-dimensional problem. In the corresponding 3-dimensional problem one considers a rigid sphere with radius $R$ immersed in the fluid. Here the 2-dimensional case of interest is treated in parallel with the well-known 3-dimensional case in order to see the analogies and differences. The symbol $D$ is used to indicate the dimension.

Far away from the immersed particle the velocity field approaches the unperturbed velocity

$$
v_{\mu}^{0}=\Gamma_{\mu \nu} r_{v}+\varepsilon_{\mu \lambda \nu} \omega_{\lambda} r_{v}
$$

where $\boldsymbol{r}=0$ is a point in the center or on the axis of the particle. Cartesian components are denoted by Greek subsripts, the summation convention is used for them. The deformation rate tensor $\Gamma_{\mu \nu}={ }^{\nabla_{\mu} v_{v}^{0}}$ is the symmetric traceless part of the unperturbed flow field, the symbol ' $\cdots$ ' indicates the symmetric traceless part of a tensor. For two vectors $\boldsymbol{a}$ and $\boldsymbol{b}$, e.g., the symmetric traceless part of the dyadic is $\overline{a_{\mu} b_{v}}=$ $(1 / 2)\left(a_{\mu} b_{v}+a_{v} b_{\mu}\right)-(1 / D) a_{\lambda} b_{\lambda} \delta_{\mu \nu}$, and $\delta_{\mu \nu}$ stands for the $D$-dimensional unit tensor. In the following, all lengths are expressed in units of the radius $R$, hence $\boldsymbol{r}$ and $\nabla$ are dimensionless quantities and the scaled velocity $v$ has the dimension $1 /$ time. The velocity field is written as $v=v^{0}+v^{1}$ where $v^{1}$ is the distortion of the velocity field due to the presence of the inclusion.

For a week stationary flow, the momentum conservation equation reduces to

$$
\nabla_{\mu} p+\nabla_{v}{ }^{\prime} \overline{p_{v \mu}}=0,
$$

where $p$ is the scalar pressure. In the hydrodynamic regime one has

$$
{ }^{p_{v \mu}}=-2 \eta{ }^{\prime} \overline{\nabla_{\nu} v_{\mu}} ',
$$

where $\eta$ is the viscosity of the fluid. Insertion of (3) into (2) and use of the incompressibility condition $\nabla_{v} v_{v}=0$ leads to

$$
\eta \Delta v_{\mu}=\nabla_{\mu} p
$$

$\Delta=\nabla_{v} \nabla_{v}$ is the Laplace operator in $D$ dimensions. Due to $\nabla_{v} v_{v}=0$, this equation implies

$$
\Delta p=0 .
$$

Equations (4) and (5) have to be solved with the appropriate boundary conditions. For the velocity these are $v_{v} \rightarrow v_{v}^{0}$ for $r \rightarrow \infty$, and for $r=1$, corresponding to the surface of the inclusion,

$$
v_{\mu}=\varepsilon_{\mu \lambda \nu} \Omega_{\lambda} r_{v}
$$

Here $\Omega_{\lambda}$ is a cartesian component of the angular velocity of the inclusion. The cases of a freely rotating inclusion with $\boldsymbol{\Omega}=\omega$ and of a non-rotating one with $\boldsymbol{\Omega}=0$ are considered in the following. The boundary condition for the pressure is $p \rightarrow 0$ for $r \rightarrow \infty$.

\subsection{Cartesian Multipole Potential Tensors}

The solution of the velocity and the pressure can conveniently be formulated in terms of Cartesian multipole potentials which, for $r \neq 0$, are solutions of the Laplace equation. For $D=3$ these tensors were already used by Maxwell. For dimensions $D \geq 2$ the multipole potential tensors of rank $\ell$ are defined by [9]

$$
X_{\mu_{1} \ldots \mu_{\ell}}^{(\ell)}=(-1)^{\ell} \nabla_{\mu_{1}} \ldots \nabla_{\mu_{\ell}} X^{(0)},
$$

where $X^{(0)}$ is the isotropic solution of the Laplace equation. With $X^{(0)}=\ln \left(r^{-1}\right)$ for $D=2$ and $X^{0}=$ $(D-2)^{-1} r^{-(D-2)}$, for $D \geq 3$, the first few of these tensors are

$$
\begin{aligned}
X_{\mu}^{(1)} & =r^{-D} r_{\mu}=r^{-(D-1)} \hat{r}_{\mu}, \\
X_{\mu \nu}^{(2)} & =D r^{-(D+2)} r_{\mu} r_{v}-r^{-D} \delta_{\mu v} \\
& =D r^{-D^{\prime} \hat{r}_{\mu} \hat{r}_{v}^{\prime}}, \\
X_{\mu v \lambda}^{(3)}= & D(D+2) r^{-(D+4)} r_{\mu} r_{v} r_{\lambda} \\
& -D r^{-(D+2)}\left(r_{\mu} \delta_{v \lambda}+r_{v} \delta_{\lambda v}+r_{\lambda} \delta_{\mu v}\right) \\
= & D(D+2) r^{-(D+1)}{ }^{\prime} \hat{r}_{\mu} \hat{r}_{v} \hat{r}_{\lambda}
\end{aligned}
$$

The unit vector parallel to $\boldsymbol{r}$ is denoted by $\hat{\boldsymbol{r}}$.

\subsection{Pressure and Velocity}

In the absence of an inclusion the pressure $p$ is constant and one puts $p=0$. Due to symmetry considerations, the scalar pressure distorted by the presence of the isotropic inclusion is proportional to the scalar $\Gamma_{\mu v} X_{\mu \nu}^{(2)}$. Insertion of this ansatz into (5) implies that the proportionality coefficient must be constant. Similarly, the distortion $v_{\mu}^{1}$ of the velocity field can only be a linear combination of terms proportional to $\Gamma_{\mu \nu} X_{\nu}^{(1)}$ and $X_{\mu \nu \lambda}^{(3)} \Gamma_{v \lambda}$ as well as $\varepsilon_{\mu \lambda \nu} \Omega_{\lambda} X_{v}^{(1)}$ and 

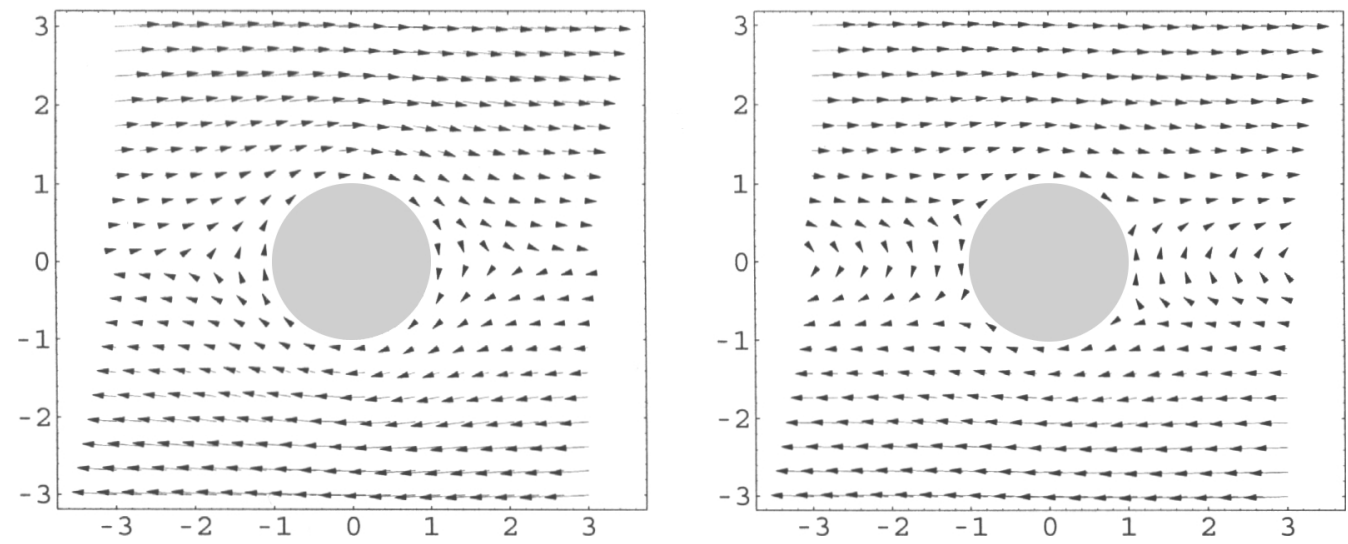

Fig. 1. The distorted flow field for a rotating (left) and a non-rotating cylinder (right).

$\varepsilon_{\mu \lambda \nu} \omega_{\lambda} X_{v}^{(1)}$. The scalar proportionality coefficients can still be functions of $r$. Insertion of this ansatz into (4), use of the above mentioned expression for $p$ and of the boundary conditions leads to

$$
\begin{aligned}
v_{\mu}^{1}= & -\Gamma_{\mu \nu} X_{v}^{(1)}+\frac{1}{2 D} X_{\mu \nu \lambda}^{(3)} \Gamma_{v \lambda} \\
& +\varepsilon_{\mu \lambda \nu}\left(\Omega_{\lambda}-\omega_{\lambda}\right) X_{v}^{(1)}, \\
p= & -\frac{D+2}{D} \eta \Gamma_{\mu \nu} X_{\mu \nu}^{(2)} .
\end{aligned}
$$

The total velocity field $\boldsymbol{v}=\boldsymbol{v}^{0}+\boldsymbol{v}^{1}$ can be written as

$$
\begin{aligned}
v_{\mu}= & \left(1-r^{-(D+2)}\right) \Gamma_{\mu \nu} r_{\nu} \\
& +\frac{D+2}{D}\left(r^{-(D+2)}-r^{-D}\right) r_{\mu} \hat{r}_{\nu} \Gamma_{\nu \lambda} \hat{r}_{\lambda} \\
& +\left(1-r^{-D}\right) \varepsilon_{\mu \lambda \nu} \omega_{\lambda} r_{v}+r^{-D} \varepsilon_{\mu \lambda \nu} \Omega_{\lambda} r_{\nu} .
\end{aligned}
$$

The relations (8) and (9) were used. The expression for $p$ is equivalent to

$$
p=-(D+2) \eta r^{-D} \hat{r}_{v} \Gamma_{\nu \lambda} \hat{r}_{\lambda} .
$$

For the case $D=3$ and a rotating sphere with $\boldsymbol{\Omega}=\omega$, the results $(10-13)$ agree with those found in [3]. The solution for the velocity field published by Einstein in 1906 [1] contained an error. The correct solution was first obtained by L. Hopf, as reported by Einstein 5 years later [2] when he corrected the expression for the effect of inclusions on the effective viscosity.

Here the case $D=2$ is of special interest. In Fig. 1 the flow field is shown for a non-rotating cylinder (right graph) and for the case where the cylinder rotates with the vorticity (left graph). In Fig. 2 corresponding velocity profiles are depicted. Cross sections through the velocity field $v_{x}(x, y)$ are shown for $x=0,1.0$ and 2.0. The thick and thin curves pertain to the non-rotating and the rotating cylinder, respectively. The unperturbed velocity profile with the shear rate $\gamma=1$, in reduced units, is indicated by the dashed straight lines. Clearly the inclusion-induced distortion of the velocity profile is more significant for the the non-rotating inclusion.

Smoothed Particle Dynamics computer simulations [8] yield pressure and velocity fields which agree with those calculated here for a fluid with a Newtonian viscosity. The numerical calculations can be and have been extended to non-Newtonian fluids, in particular to a co-rotational Maxwell model [10], as well as to higher volume fractions.

\section{Effective Viscosity}

\subsection{General Remarks}

The presence of inclusions modifies the velocity field, and as a consequence the effective viscosity $\eta_{\text {eff }}$ differs from the viscosity $\eta$ of the pure fluid. Physical intuition says $\eta_{\text {eff }}>\eta$. For dilute suspensions, the case originally considered by Einstein and also treated here, the effect of $N>1$ inclusions is assumed to be additive. Hence it suffices to study the influence of a single inclusion and to multiply the resulting difference $\eta_{\text {eff }}-\eta$ by the factor $N$. For one isotropic inclusion one expects

$$
\eta_{\mathrm{eff}}=\eta\left(1+Z V_{R} / V\right)
$$

with a numerical coefficient $Z$, which has to be calculated. The quantities $V$ and $V_{R}$ are the $D$-dimensional volumes of the fluid and of the inclusion. In particular 


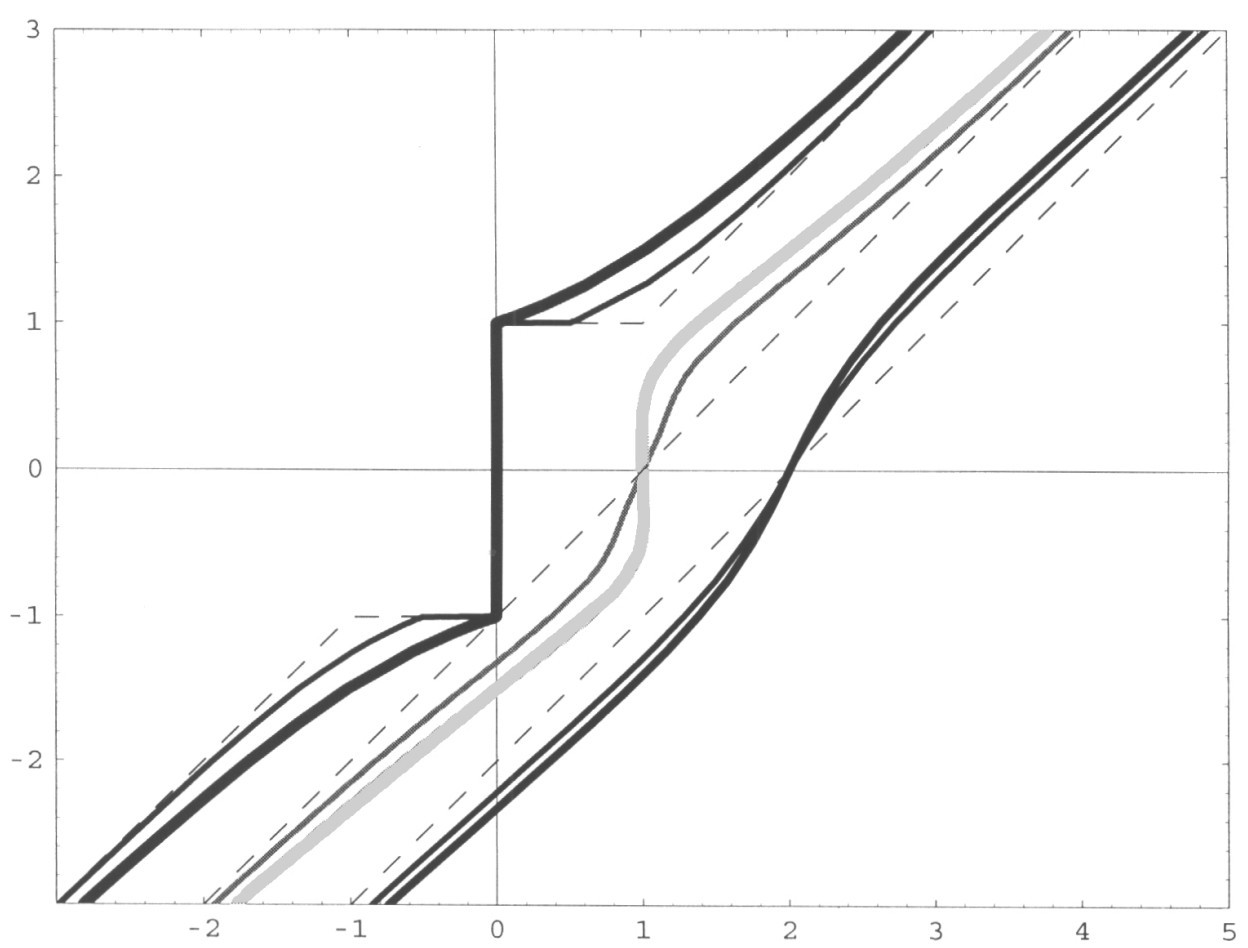

Fig. 2. The velocity profile for rotating (thin curves) and non-rotating cylinders (thick curves) for cross sections through the velocity field $v_{x}(x, y)$ at $x=0.0,1.0,2.0$. The dashed straight lines indicate the unperturbed velocity profile with the shear rate $\gamma=1$, in reduced units.

one has

$$
V_{R}=\frac{1}{D} R^{D} \int \mathrm{d}^{(D-1)} \hat{\boldsymbol{r}}
$$

where it is understood that the integration $\mathrm{d}^{(D-1)} \hat{\boldsymbol{r}}$ has to be taken over the $D$-dimensional unit sphere. Thus one has $V_{R}=\pi R^{2}$ and $V_{R}=(4 \pi / 3) R^{3}$ for $D=2$ and $D=3$, respectively. In the following, $V$ is taken as the volume of a concentric $D$-dimensional sphere with $r>>1$ for computational convenience, thus $V=r^{D} V_{R}$. The shape of $V$ does not matter for an inclusion small compared to the size of a container.

For a suspension with $N$ inclusions, (14) leads to

$$
\eta_{\mathrm{eff}}=\eta(1+Z \varphi), \varphi=N\left(V_{R} / V\right)=n V_{R}
$$

Here $\varphi$ is the volume fraction (also called packing fraction) of the inclusions, $n=N / V$ is their number density.

One might assume that the effective viscosity could simply be inferred from the volume average of the friction pressure tensor ' $\overline{p_{\mu v}}$,, $\mathrm{cf}$. (3). The integration over the angles involved in the volume integral of $\nabla_{\nu} v_{\mu}^{1}$ vanishes, and one has $\int{ }^{\prime} \overline{\nabla_{v} v_{\mu}}{ }^{\prime} \mathrm{d}^{D} r=\int \bar{\nabla}_{\nu} v_{\mu}^{0}=$ $\left(V-V_{R}\right) \Gamma_{\mu \nu}$. The magnitude of the volume average of ' $\overline{p_{\mu v}}$ ' is smaller in the presence of the inclusion than without it, thus the ratio between this quantity and $\Gamma_{\mu \nu}$ cannot be used to compute the effective viscosity. However, it is noticed that for a plane Couette flow (with asymptotic flow in $x$-direction and the gradient in $y$-direction) the effective shear rate $\gamma_{\text {eff }}$, inferred from the volume average of the total velocity gradient, is related to the shear rate $\gamma=\partial v_{x}^{0} / \partial y$ of the unperturbed flow by

$$
\gamma_{\mathrm{eff}}=\left(1-\left(V_{R} / V\right)\right) \gamma
$$

This relation plays an important role in the calculation of the effective viscosity via the route proposed by Einstein $[1,2]$, where $\eta_{\text {eff }}$ is inferred from the entropy production divided by $\gamma_{\mathrm{eff}}^{2}$.

\subsection{The Einstein Route via Entropy Production}

The entropy production density is proportional to the heat of friction per volume $-\left(\nabla_{v} v_{\mu}\right)^{\prime} \overline{p_{v \mu}}$ '. In 
the absence of an inclusion this quantity is equal to $2 \eta \Gamma_{v \mu} \Gamma_{v \mu}=\eta \gamma^{2}$. Thus one defines $\eta_{\mathrm{eff}}$ as the ratio of

$$
\begin{aligned}
\Theta & =-V^{-1} R^{D} \int\left(\nabla_{v} v_{\mu}\right) \cdot \overrightarrow{p_{v \mu}} \mathrm{d}^{D} r \\
& =\eta V^{-1} R^{D} \int\left(\nabla_{v} v_{\mu}\right)\left(\nabla_{v} v_{\mu}+\nabla_{\mu} v_{v}\right) \mathrm{d}^{D} r
\end{aligned}
$$

and $\gamma_{\mathrm{eff}}^{2}$.

The velocity field is a sum of a deformational part involving the asymptotic deformation rate $\Gamma_{\mu \nu}$ and a rotational part involving the vorticity and the angular velocity of the particle. Likewiswe the quantity $\Theta$ is a sum of deformational contribution $\Theta^{\text {def }} \sim \Gamma_{\nu \mu} \Gamma_{\nu \mu}$ and a rotational contribution $\Theta^{\text {rot }} \sim\left(\omega_{\mu}-\Omega_{\mu}\right)\left(\omega_{\mu}-\Omega_{\mu}\right)$. Both contributions can be treated independently.

It is possible to transform the volume integral in (18) into a surface integral which is particularly convenient for the deformational contribution. An integration by parts of the first expression in (18) and use of (4) yields $\Theta=-V^{-1} R^{D} \int n_{v} v_{\mu} \bar{p}_{v \mu} \mathrm{d}^{(D-1)} f-$ $V^{-1} R^{D} \int v_{\mu} \nabla_{\mu} p \mathrm{~d}^{D} r$, where $\boldsymbol{n}$ is the outer normal of the volume in which the fluid is contained, and $\mathrm{d}^{(D-1)} f$ stands for the surface element. The remaining volume integral is transformed into the surface integral $-V^{-1} R^{D} \int n_{\mu} v_{\mu} p \mathrm{~d}^{(D-1)} f$, where $\nabla_{\mu} v_{\mu}=0$ has been used. The outer normal is $\boldsymbol{n}=\hat{\boldsymbol{r}}$ for the outer surface corresponding to $r>>1$, and $\boldsymbol{m}=-\hat{\boldsymbol{r}}$ for the inner surface at $r=1$. When only the outer surface is taken into account, one finds

$$
\begin{array}{r}
\Theta=V^{-1} R^{D} r^{D} \int\left[\eta\left(r^{-2} v_{\mu} \nabla_{\mu}\left(r_{v} v_{v}\right)+v_{\mu}\left(r^{-1} v_{\mu}\right)^{\prime}\right)\right. \\
\left.-r^{-2} r_{v} v_{v} p\right] \mathrm{d}^{(D-1)} \hat{\boldsymbol{r}},
\end{array}
$$

where the prime' denotes the differentiation with respect to $r$. There is no contribution to $\Theta^{\text {def }}$ from the integral over the inner surface, since the deformational part of the velocity vanishes for $r=1$, cf. (12). The first and the last term in (19) vanish for the rotational part of the velocity. The second term leads an expression proportional to $\omega_{\mu}\left(\omega_{\mu}-\Omega_{\mu}\right)$. The only contribution from the integral over the inner surface is proportional to $-\Omega_{\mu}\left(\omega_{\mu}-\Omega_{\mu}\right)$. Thus for both limiting cases, $\boldsymbol{\Omega}=0$ and $\boldsymbol{\Omega}=\omega$, there is no contribution at all from the integral over the inner surface.

Starting from the velocity field and the pressure given above, the quantities $\Theta^{\text {def }}$ and $\Theta^{\text {rot }}$ can now be calculated. The integrals over the angles $\int \ldots \mathrm{d}^{(D-1)} \hat{\boldsymbol{r}}$ are expressed in terms of angular averages defined by

$$
\langle\ldots\rangle=\left(\int d^{(D-1)} \hat{\boldsymbol{r}}\right)^{-1} \int \ldots \mathrm{d}^{(D-1)} \hat{\boldsymbol{r}} .
$$

Use is made of the fact that angular averages involving two irreducible tensors of different rank vanish. For integrals containing tensors of equal rank $\ell$, e.g. $\ell=1$ and $\ell=2$, one has

$$
\begin{aligned}
& \left\langle r_{\mu} r_{v}\right\rangle=\frac{1}{D} \delta_{\mu v}, \\
& \left\langle\overline{r_{\mu} r_{v}} \cdot \overline{r_{\lambda} r_{\kappa}}\right\rangle=\frac{1}{D(D+2)} \Delta_{\mu v, \lambda \kappa},
\end{aligned}
$$

where the isotropic forth rank tensor $\Delta_{\ldots}$ is defined by [9]

$$
\Delta_{\mu v, \lambda \kappa}=\frac{1}{2}\left(\delta_{\mu \lambda} \delta_{v \kappa}+\delta_{\mu \kappa} \delta_{v \lambda}\right)-\frac{1}{D} \delta_{\mu v} \delta_{\lambda \kappa} .
$$

This tensor has the properties $\Delta_{\mu \lambda, \lambda \kappa}=\frac{(D-1)(D+2)}{2 D} \delta_{\mu \kappa}$ and $\Delta_{\mu v, \mu v}=\frac{(D-1)(D+2)}{2}$. Furthermore, one has ${ }^{\prime} \hat{r}_{\mu} \hat{r}_{v} ' \hat{r}_{\mu} \hat{r}_{v}=\frac{D-1}{D}$.

When terms which vanish in the limit $r \rightarrow \infty$ are disregarded, the first term in the surface integral (19) yields the contribution $\eta\left(2-4 V_{R} / V\right) \Gamma_{\mu \nu} \Gamma_{\mu \nu}$ to $\Theta^{\text {def }}$. The second and third terms give contributions $D \eta\left(V_{R} / V\right) \Gamma_{\mu \nu} \Gamma_{\mu \nu}$ and $2 \eta\left(V_{R} / V\right) \Gamma_{\mu \nu} \Gamma_{\mu \nu}$, respectively. Thus one obtains

$$
\Theta^{\mathrm{def}}=2 \eta \Gamma_{\mu \nu} \Gamma_{\mu \nu}\left[1+\left(\frac{D}{2}-1\right) \frac{V_{R}}{V}\right] .
$$

Division of $\Theta^{\text {def }}$ by $\gamma_{\text {eff }}^{2}$ with the effective shear rate given in (17) and neglect of terms non-linear in $V_{R} / V$ now leads to an effective viscosity of the form (16) with the factor $Z$ given by

$$
Z=\frac{D}{2}+1
$$

This result applies to an inclusion which freely rotates with an angular velocity equal to the vorticity. For $D=3$ the value $Z=5 / 2$, first presented by Einstein in 1911 [2], is recovered. For $D=2$ one has $Z=2$.

The calculation of the rotational part of $\Theta$ yields

$$
\Theta^{\mathrm{rot}}=2 D \eta\left(\omega_{\mu}-\Omega_{\mu}\right)\left(\omega_{\mu}-\Omega_{\mu}\right) \frac{V_{R}}{V} .
$$


For the case of a non-rotating inclusion corresponding to $\boldsymbol{\Omega}=0$ in a plane Couette flow with the asymptotic shear rate $\gamma$ one has $\omega^{2}=\gamma / 4$. The total entropy production then is

$$
\Theta=\eta \gamma^{2}\left(1+(D-1) \frac{V_{R}}{V}\right)
$$

Thus the factor $Z$ in the expression for the effective viscosity is now given by

$$
Z=D+1 \text {. }
$$

This value is larger than (24). The difference between the effective viscosity of a fluid containing nonrotating spheres $(D=3)$ and the case of freely rotating spheres is $(3 / 2) \eta\left(V_{R} / V\right)$. This has previously been noticed in connection with the influence of a magnetic field on the viscosity of dilute ferro-fluids [11-13]. For $D=2$ the corresponding expression is $\eta\left(V_{R} / V\right)$.

Of course it is desireable to calculate the effective viscosity by a more direct method where this quantity is inferred from the ratio of the relevant component of an effective stress tensor and the effective shear rate. For $D=3$ and $\boldsymbol{\Omega}=\omega$ such a computation is found in [3]. For the more general case studied here a moderate modification of that method is presented next.

\subsection{The Route via an Effective Stress Tensor}

At the surface of a fluid (or solid) substance a force $F$ with $F_{v} \mathrm{~d}^{(D-1)} f=-n_{\lambda} p_{\lambda \nu} \mathrm{d}^{(D-1)} f$ is needed in order to maintain mechanical equilibrium. Here $p_{\lambda v}=$ $p \delta_{\lambda v}+{ }^{\prime p_{\lambda v}}$ ' are the components of the total pressure tensor. The effective stress tensor $\sigma_{\mu \nu}^{\text {eff }}$ is obtained as an average of $r_{\mu} F_{\nu}$ according to $V \sigma_{\mu \nu}^{\text {eff }}=$ $\int r_{\mu} F_{\nu} \mathrm{d}^{(D-1)} f$ [14]. Here $V$ is the volume enclosed by the surface considered. Again a concentric $D$ dimensional sphere with radius $r>>1$ is chosen where $n=\hat{\boldsymbol{r}}$. Then one has

$$
\sigma_{\mu \nu}^{\mathrm{eff}}=-V^{-1} R^{D} \int r_{\mu} \hat{r}_{\lambda} p_{\lambda v} \mathrm{~d}^{D-1} \hat{\boldsymbol{r}}
$$

Use of (3) leads to

$$
\begin{aligned}
\sigma_{\mu \nu}^{\text {eff }}= & -V^{-1} R^{D} \int\left[\eta r _ { \mu } \left(r^{-1} \nabla_{v}\left(r_{\lambda} v_{\lambda}\right)\right.\right. \\
& \left.\left.+r\left(r^{-1} v_{v}\right)^{\prime}\right)-r_{v} \hat{r}_{v} p\right] \mathrm{d}^{(D-1)} \hat{\boldsymbol{r}}
\end{aligned}
$$

This expression is evaluted with $v$ and $p$ given above and with the help of the relations (21). Again terms which vanish in the limit $r \rightarrow \infty$ are disregarded. The result is

$$
\begin{aligned}
\sigma_{\mu \nu}^{\mathrm{eff}} & =2 \eta \Gamma_{\mu v}\left(1+\frac{D}{2} \frac{V_{R}}{V}\right) \\
& +\eta D \frac{V_{R}}{V} \varepsilon_{\mu v \lambda}\left(\omega_{\lambda}-\Omega_{\lambda}\right)
\end{aligned}
$$

For an inclusion with an angular velocity which matches the vorticity, division of the effective stress by the effective shear rate leads to an effective viscosity with the factor $Z=1+D / 2$, just as above.

Next the case $\boldsymbol{\Omega}=0$ is considered. Due to $2 \varepsilon_{\mu \nu \lambda} \omega_{\lambda}=\nabla_{\mu} v_{v}^{0}-\nabla_{v} v_{\mu}^{0}$, now (30) is equivalent to

$$
\begin{aligned}
\sigma_{\mu \nu}^{\mathrm{eff}}= & \eta\left(1+\frac{D}{2} \frac{V_{R}}{V}\right)\left(\nabla_{\mu} v_{v}^{0}+\nabla_{\nu} v_{\mu}^{0}\right) \\
& +\eta \frac{D}{2} \frac{V_{R}}{V}\left(\nabla_{\mu} v_{v}^{0}-\nabla_{\nu} v_{\mu}^{0}\right)
\end{aligned}
$$

For a plane Couette flow in $x$-direction with the gradient in $y$-direction (31) yields the effective shear stress $\sigma=\sigma_{y x}^{\text {eff }}=\eta\left(1+D V_{R} / V\right)$. Division of this expression by the effective shear rate leads to an effective viscosity with $Z=1+D$, again in accord with the corresponding result stated above.

Notice that, in general, the effective stress tensor is symmetric only when the angular velocity of the inclusion is equal to the (asymptotic) vorticity. The antisymmetric part of the tensor is associated with the torque

$$
T_{\lambda}=V \varepsilon_{\lambda \mu \nu} \sigma_{\mu \nu}^{\mathrm{eff}}
$$

excerted by the fluid on the inclusion. For the case where the fluid is at rest far away from the inclusion, the torque $T$, as inferred from (30), is given by

$$
\boldsymbol{T}=-2 D \eta V_{R} \boldsymbol{\Omega} .
$$

Thus, as a by-product, the rotational friction coefficient $8 \pi \eta R^{3}$ is recovered for $D=3$, [3]. For $D=2$, the corresponding expression is $4 \pi \eta R^{2}$.

So far, it has been demonstrated that the calculation of the effective viscosity via both routes yields identical results for non-rotating and for vorticity adapted rotating inclusions. For arbitrary values of the angular velocity the contribution proportional to $\Omega_{\mu}\left(\omega_{\mu}-\Omega_{\mu}\right)$ in the entropy production (25), which stems from the integration of the inner surface, however, has to be disregarded in the prescription for the calculation of $\eta^{\text {eff }}$, when the entropy production route should give the same result as via the effective stress tensor route. 


\section{Concluding Remarks}

The velocity and pressure have been calculated for rotating and non-rotating $D$-dimensional isotropic inclusions. The ensuing effective viscosity of a dilute dispersion was evaluted via two routes which yielded equivalent results. The method involving an effective stress tensor is apt to treat the problem of elliptical or ellipsoidal non-rotating inclusions in a Newtonian fluid in analogy to the calculation of the viscosity of perfectly oriented nematic liquid crystals by an affine transformation [15]. Furthermore, it is desireable to

[1] A. Einstein, Ann. Phys. 19, 289 (1906).

[2] A. Einstein, Ann. Phys. 34, 591 (1911).

[3] L. D. Landau and E. M. Lifschitz, Lehrbuch der Theoretischen Physik, Bd. VI: Hydrodynamik, Akademie Verlag, Berlin 1966.

[4] R. G. Larson, The Structure and Rheology of Complex Fluids, Oxford University Press, Oxford, UK 1999.

[5] B. N. J. Perrson, Sliding Friction, Springer, Berlin 1999.

[6] H. Krause and C. Schroelkamp, Wear 120, 353 (1987).

[7] J. Ahlström and B. Karlsson, Wear 232, 1 (1999).

[8] M. Ellero, M. Kröger, and S. Hess (to be published).

[9] S. Hess, Z. Naturforsch. 23a, 1095 (1968).

[10] M. Ellero, M. Kröger, and S. Hess, J. Non-Newtonian Fluid Mech. 5, 35 (2002). treat the inclusion problem also for non-Newtonian fluids along the lines presented here. Analytic results can provide an useful test for Smoothed Particle Dynamics (SPD) and also for Non-Equilibrium Molecular Dynamics (NEMD) computer simulations, cf. $[8,10,16]$.

\section{Acknowledgements}

This work was initiated by the DFG project HE 1100/7-1; financial support by the Deutsche Forschungsgemeinschaft is gratefully acknowledged. I thank Marco Ellero, Patrick Ilg, Martin Kröger and Igor Stankovic for helpful dicussions.

[11] W. F. Hall and S. N. Busenberg, J. Chem. Phys. 51, 137 (1969).

[12] M. I. Shliomis, Sov. Phys. JETP 34, 1291 (1972).

[13] P. Ilg, M. Kröger, S. Hess, and A. Y. Zubarev, Phys. Rev. E 67, 061401 (2003).

[14] L. D. Landau and E. M. Lifschitz, Lehrbuch der Theoretischen Physik, Bd. VII, Elatizitätstheorie, Akademie Verlag, Berlin 1965.

[15] D. Baalss and S. Hess, Phys. Rev. Lett. 57, 86 (1986); Z. Naturforsch. 43a, 662 (1988).

[16] I. Stankovic, S. Hess, and M. Kröger, Phys. Rev. E 69, 021509 (2004). 